\title{
REPENSAR EL FUTURO DEL AGUA EN NUESTRO PLANETA
}

\section{ARTÍCULO DE REVISIÓN}

DIAS, Deusira Nunes Di Lauro ${ }^{1}$

DIAS, Adailton Di Lauro ${ }^{2}$

DIAS, Deusira Nunes Di Lauro. DIAS, Adailton Di Lauro. Repensando el futuro del agua en nuestro planeta. Revista Científica Multidisciplinar Núcleo do Conhecimento. año 04, Ed. 08, Vol. 06, págs. 19-32. agosto de 2019. ISSN: 24480959

\section{RESUMEN}

Considerado el líquido más importante, precioso e indispensable para la supervivencia de la vida humana en la Tierra, el agua ha sido motivo de preocupación constante, porque la forma en que la sociedad se ha apropiado y utilizado los recursos hídricos en el planeta Comprometidos con la calidad, así como el acceso de muchas comunidades a este recurso vital para el mantenimiento de la vida. Este trabajo pretende analizar y discutir estrategias que garanticen la preservación y el uso sostenible del agua en el planeta, abordando que, en algunas regiones de Brasil y del mundo, ya están con extrema escasez de agua y, según algunos autores, este problema tiende a aumentar cada vez más debido a varios factores, y los principales son el rápido crecimiento de la población y la gran demanda de alimentos en el mundo.

1 Graduado en el curso de cartas de la Universidad Estatal de Bahía - UNEB Campus X; Postgraduado en Portugués por la Universidad de Vale do Cricaré y un estudiante regular de la maestría en educación de la Universidad e Internacional Unigrendal en línea.

${ }^{2}$ Graduado en letras por Uneb, graduado en inglés por Unimes, postgraduado de los Colegios Integrados de Jacarepaguá (FIG), y estudiante regular de la maestría en educación en la Universidad Internacional Unigrendal en línea. 
Las leyes que rigen los recursos hídricos se están ajustando cada vez más para preservar los recursos hídricos y todo el medio ambiente. El Brasil ha demostrado que es necesario preservar, por mucho que quede mucho por hacer para minimizar los impactos causados por el hombre en el medio ambiente.

Palabras clave: agua, escasez, preservación, sostenibilidad.

\section{INTRODUCCIÓN}

La humanidad ha logrado, especialmente en este siglo, innumerables logros nunca imaginados por la ciencia y la tecnología. Sin embargo, el problema del agua y el mantenimiento de la vida en el planeta ha llevado a gran parte de la población mundial a preguntarse qué hacer para garantizar que todos tengan acceso al agua, pero no siempre tiene una respuesta, porque el ser humano no es siempre o casi nunca ser $D$ Explicar la necesidad de preservar este preciado producto.

Esta preocupación en el futuro no ocurre por casualidad, después de todo, los datos sobre la distribución, reserva y disponibilidad de agua en el planeta evidencian la necesidad de buscar alternativas para la preservación, como dice VICTORINO:

Se sabe que casi toda la superficie del planeta Tierra está cubierta por agua: desde los océanos, ríos y lagos, arroyos y Sanghas. Agua de casquetes polares, agua de lluvia, mucho, mucha agua... Pero en realidad no todo es azul (el color que cubre la tierra - debido al aguacuando se ve desde el espacio), porque toda el agua en el planeta (1.37 mil millones de $\mathrm{km} \mathrm{3}$ ) está constituida básicamente de dos tipos: agua salada de los mares y agua dulce de ríos, lagos y subsuelo. Sin embargo, el $97 \%$ del total está ocupado por agua salada, inadecuada para el consumo. Con el llamado "ciclo del agua" (proceso de evaporación y retorno del agua a la superficie en forma de lluvia, nieve, granizo o helada), el agua se recompone de varias maneras dando vida a las plantas y generando varios Biomms siguiendo el curso natural de la vida. La naturaleza es sabia y sólo porque cuando el agua escapa a la 
atmósfera en forma de evaporación, la transpiración se compensa con precipitaciones. Esta diferencia entre el volumen de agua que cae y el volumen de agua que se evapora es de unos 45000 kilómetros cúbicos por año, que, en teoría, el ser humano podría gastar. Sólo el $20 \%$ de este total está disponible. El agua utilizable está en ríos, lagos, agua de lluvia y agua subterránea. Sin embargo, todos juntos corresponden a sólo el $1 \%$ del volumen de agua dulce (VICTORINO, 2007, p. 16).

Figura 1-gráfico-"distribución de agua en la Tierra"

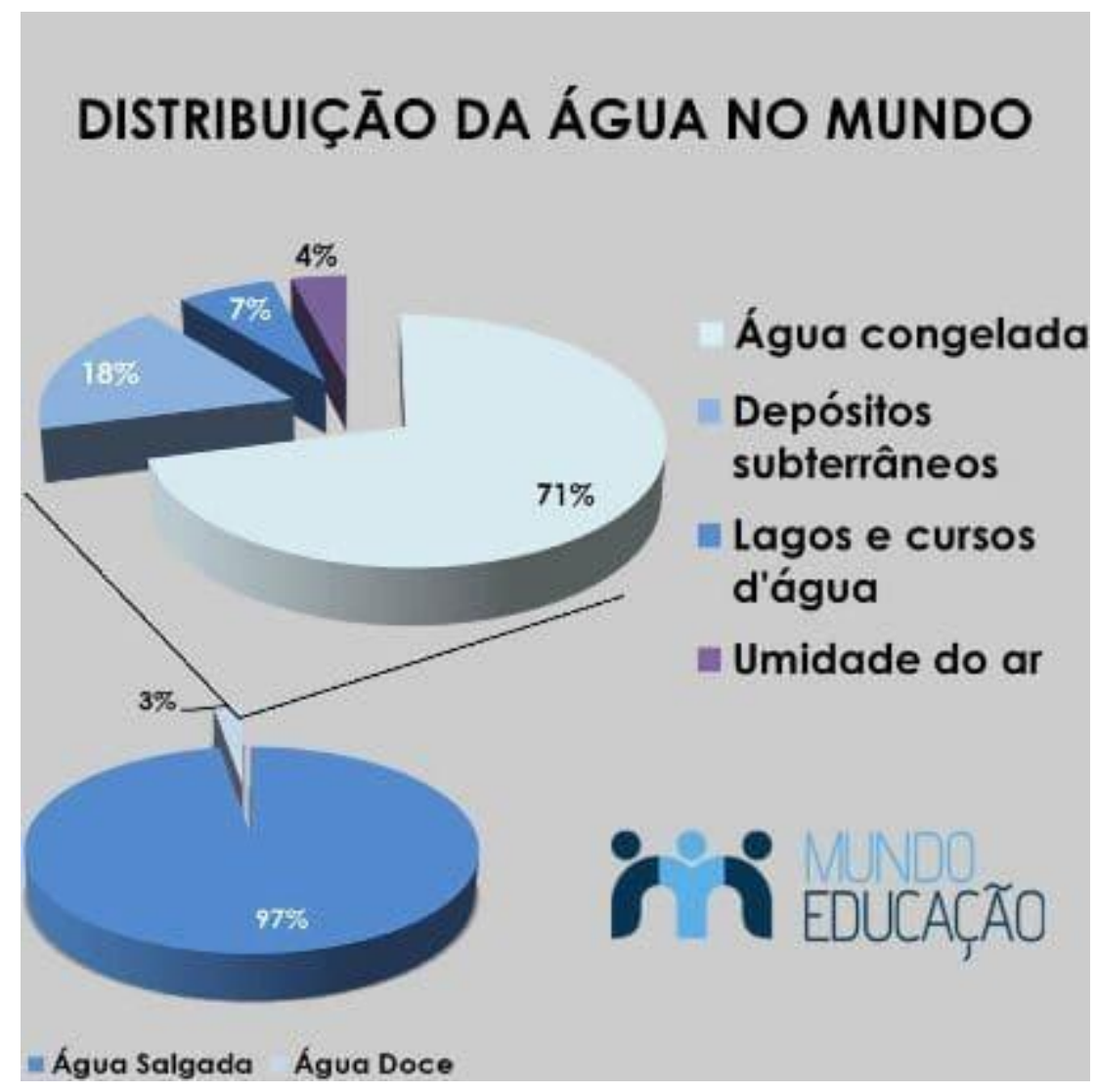

(Fuente: Sitio de Educación Mundial)

Al analizar el gráfico anterior, se observa que la cantidad de agua disponible para su uso es insuficiente, ya que:

Si juntamos 1,5 litros de agua, como la encontramos en el planeta, y la dividimos proporcionalmente, la cantidad de agua dulce disponible 
equivaldría a una sola caída insignificante. Y para complicar, este pequeño resto está cada vez más contaminado, especialmente en las grandes ciudades, debido al aumento de la población mundial que, sólo en el siglo pasado se hizo tres veces mayor, lo que causó el aumento de fábricas, más residuos y más riego En las cosechas. Según el Banco Mundial, alrededor de 80 países se enfrentan hoy a problemas de suministro. La situación más crítica es en Asia, donde el $60 \%$ de la población vive con sólo el $32 \%$ del agua dulce disponible (VICTORINO, p. 17).

Al analizar todos estos datos, es posible evaluar la gravedad de los impactos causados por las acciones del hombre al medio ambiente y, específicamente al agua, que se considera un recurso vital para la vida en la Tierra. Muchos países ya han adoptado posturas de contención y preservación a través de tratados, acuerdos, con el fin de minimizar esos impactos. Sin embargo, una parte considerable de la población mundial sigue ignorando que el futuro está amenazado y que habrá un cambio, no sólo de mentalidad, sino también de actitud hacia el uso de este recurso que se ha utilizado de manera irresponsable para larga historia.

Se sabe que la población mundial ha crecido de manera desordenada, y esto también exige un aumento considerable de la producción agrícola industrial en un intento de garantizar los alimentos para todos, lo que impone presiones cada vez más intensas sobre el medio ambiente. En vista de esto, es necesario encontrar estrategias que cancelen los impactos, ya que se espera una población de más de ocho mil millones de personas para el año 2020:65\% en zonas costeras y el 60\% en ciudades con más de 2,5 millones de personas. Además, el uso del suministro de agua aumentó enormemente en un corto período de tiempo: si bien la renovación no cambió el nivel de los recursos hídricos, de 1900 a 1995, en la contrapartida, la cantidad utilizada aumentó a seis veces más (dos veces Aumento de la población) y dos veces más desde 1975. Del total de agua consumida en el planeta, la agricultura absorbe un promedio mundial del $70 \%$ de los suministros de agua, y en los países 
subdesarrollados el porcentaje es aún mayor al registrar un aumento del 80 al 90\%, con un promedio del $20 \%$ para la industria y del $10 \%$ para usos domésticos y OR Tros.

Al analizar la situación de Brasil en este contexto, es aún más evidente que el agua no se ha utilizado de manera racional, lo que compromete y mucho el suministro y la calidad de vida en muchas regiones del país.

\section{CONSUMO DE AGUA EN EL PAÍS}

Figura 2 gráfico - consumo de agua en el país

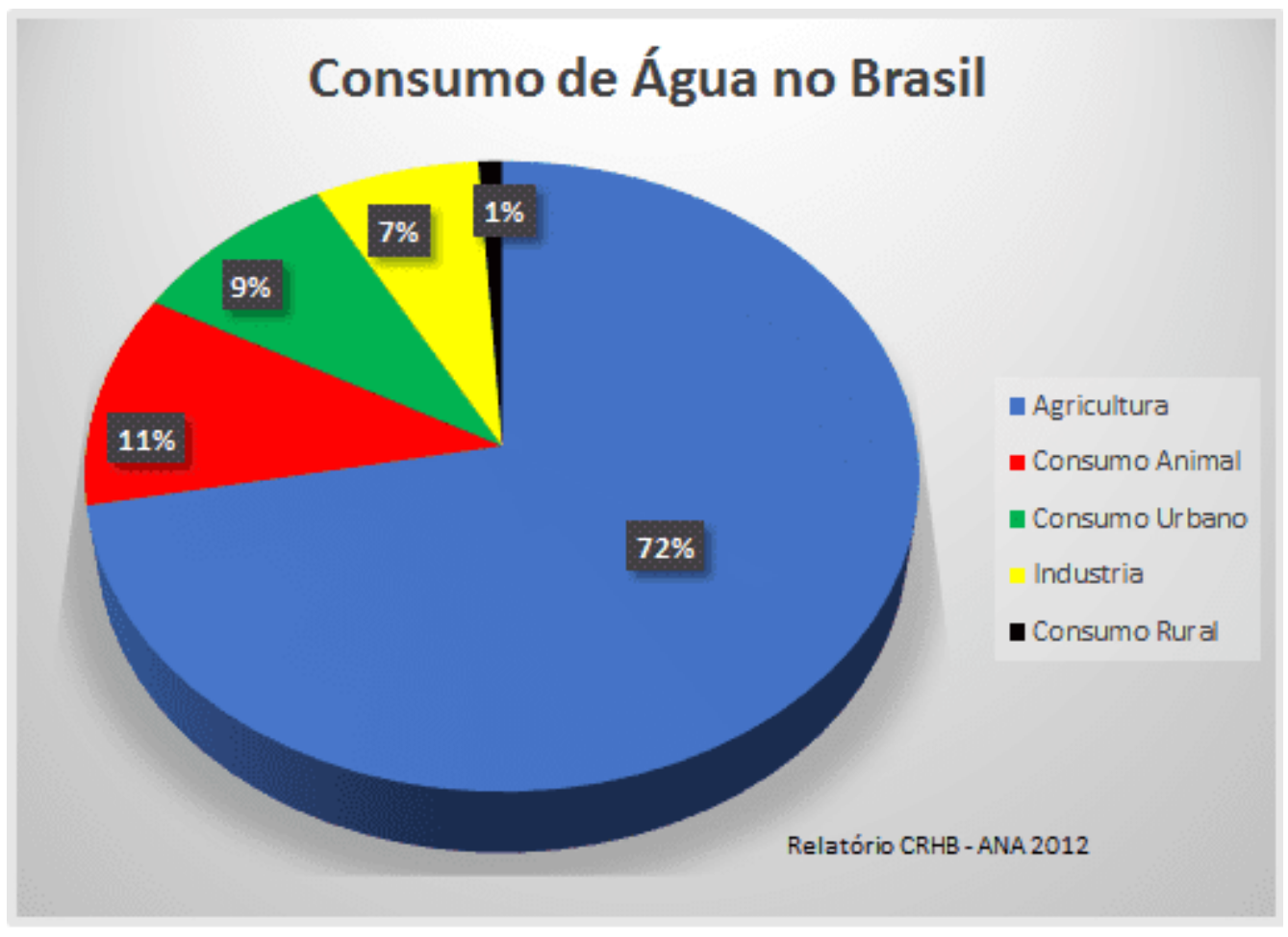

(Fuente: Consultores EOS)

El gráfico anterior señala a la agricultura como el gran villano en el consumo y desperdicio de agua en Brasil y, aunque el país es pionero en aprobar leyes que limitan el consumo e impone algunas sanciones a quienes cometen abusos en el consumo y uso del agua , también se sabe que no hay suficiente supervisión por parte de los gobernantes para asegurar que se respeten las leyes, es decir, aunque hay leyes de preservación, en la práctica está lejos de suceder. 
Hoy en día, a pesar del concepto legal de buen uso común de las personas, el agua es un recurso, para muchos, de valor económico. Esto resulta de la creciente demanda de agua para los diversos usos, lo que acompaña el desarrollo urbano e industrial de los países. Hoy en día, los grandes problemas relacionados con el agua no ocurren debido a la naturaleza, sino que surgen del mal uso con residuos e improvisación, así como de la contaminación generalizada que amenaza el medio ambiente.

El código del agua, la ley mayor que la disciplina del uso del agua en Brasil es el 10 de julio de 1934, ya tiene cierta edad, pero no es así que ya no es actual porque clasifica las categorías legales, discrimina los usos del agua y sus preceptos y , sobre todo: busca garantizar los intereses generales de la sociedad. En el antiguo código era posible encontrar el dominio privado del agua en algunos casos, pero esto fue extinguido a partir de octubre de 1988, cuando todos los cuerpos D' agua se convirtió en dominio público.

\section{LA CALIDAD DEL AGUA QUE CONSUMIMOS}

La calidad del agua disponible en el planeta es otro aspecto que también ha sido objeto de preocupación por parte de los organismos de salud de todo el mundo, ya que se evitan las enfermedades cuando se utiliza agua descontaminada. La calidad del agua ha decidido la suerte de algunas regiones de todo el mundo en carácter económico, principalmente en relación con la fabricación de cervezas. Pero no es sólo en la rama de las cervecerías que la pureza del agua es importante, también es necesario en las industrias de tapices, en la curtiduría, en la industria de la tela y en las piedras preciosas. Además, la calidad del agua determinará la calidad de vida de sus consumidores. Cuando no hay agua potable adecuada para el consumo humano, el resultado es la alta tasa de enfermedades y otros problemas derivados de ellas. Esta es una preocupación que en el pasado determinó el éxito o fracaso de las naciones más desarrolladas, como dice Célia Jurema:

Al hombre siempre le importaba el agua. Durante 4.000 años a.C., las primeras leyes que se conocían fueron códigos que regulaban el uso del agua, escritos por los sumerios. Pero no todas las civilizaciones tuvieron 
cuidado con eso. Los registros muestran que los mayas tuvieron que abandonar la ciudad de Tical, ubicada en un bosque tropical completo, donde se encontraron las ruinas de la Pirámide del Sol, porque no sabían cómo almacenar adecuadamente el agua, además de producir erosiones crecientes y grandes Se deforja porque usaban madera incluso en las estructuras internas de las columnas de sus gigantescos templos. En México, en la Península de Yucatán, el dios de la lluvia, Chac, fue venerado $y$, cada vez que el agua se aescapara, se erigieron nuevos templos con la esperanza de que el dios de las aguas salvaría a los mayas de las sequías. A menudo se encuentran, motivos del Dios de la lluvia maya en la base de las pirámides. La convivencia con los ríos trajo sabiduría a las poblaciones a lo largo del desarrollo de la humanidad, los faraones, planearon sus ciudades dejando las zonas ribereñas libres"(VICTORINO, 2007, p. 19).

Desde tiempos más remotos, el agua siempre ha sido uno de los reguladores sociales más importantes. Las estructuras de las sociedades campesinas y las comunidades de las aldeas, donde las condiciones de vida están íntimamente ligadas al suelo, se organizaron alrededor del agua. Hubo casos raros en los que todos los miembros de una comunidad estaban al mismo nivel en relación con el agua: el acceso a ella casi siempre implicaba desigualdad. Esto nos lleva a entender cómo es la relación de los seres humanos con el agua y entre sí debido al agua. El hombre, sin embargo, tardó mucho tiempo en cuidar y cuidar eficazmente la calidad del agua - muchos todavía hoy no se dieron cuenta de que, más días menos día, la escasez será una realidad, y seguir utilizando el agua indiscriminadamente es para tareas de mpl Es cómo lavar el coche con manguera o barrer la acera con Jets D'agua. Otros todavía juegan todo tipo de desperdicios en lagos, ríos y arroyos. ¿Durante cuánto tiempo la sociedad seguirá actuando de manera tan intrascendente e irresponsable? El agua, este mineral que ambos conocemos y que forma parte de nosotros, ha existido durante al menos 3.900 millones de años. El "ser racional", en pocas generaciones, ya ha logrado comprometer, no sólo la calidad, sino también la cantidad de este manantial natural. Se puede evaluar el grado de desarrollo de un pueblo por la calidad de los servicios 
de agua y saneamiento que se ofrecen a la población. La capacidad de apoyar la vida humana y la sociedad es compleja, dinámica y varía en función de la forma en que el hombre gestiona los recursos ambientales. Es extraño que los sabios sacerdotes de la Antiguedad no percibieran las correlaciones existentes entre el suministro de agua y la conservación de los bosques. En ese momento, la plantación era el privilegio de los príncipes sólo, no fue a través de sus ' brillantes ' cabezas que requeriría frentes de plantadores para reforestar las tierras devastadas:

Hace 8000 años, Brasil tenía el 9,8\% de los bosques del mundo. A principios del siglo XXI, este porcentaje cayó al $28 \%$ y, peor aún, los 64 millones de kilómetros cuadrados de bosques existentes antes de la expansión demográfica y tecnológica de los seres humanos siguen siendo menos de 15,5 millones, alrededor del $24 \%$. Esto significa que más del $75 \%$ de los bosques del mundo ya han desaparecido... Ebc

Restaurar los recursos naturales degradados es más difícil que su conservación; Y, muchos de ellos, como el agua, no pueden ser restaurados, cuando termina no hay vuelta atrás, cuando termina es para siempre. La falsa ilusión de que el agua nunca acabará siendo evidente en la naturaleza tal vez justifique, en parte, el descuido histórico de los seres humanos en sus relaciones con los recursos hídricos. Se sabe que no hay tanta agua potable disponible como el paisaje nos hace ver. Lo que realmente tenemos como agua potable es sólo el $0,03 \%$ del agua total en el planeta. Esta cantidad insignificante debe recibir todos los cuidados posibles, sin embargo, esto no es lo que vemos en casi todos los continentes, después de todo, los principales acuíferos se están agotando con una velocidad más rápida que su capacidad natural de recuperación.

\section{EL USO INDISCRIMINADO DEL AGUA Y EL COMPROMISO DE LA CALIDAD DE VIDA DE LA HUMANIDAD}

No es necesario ir demasiado lejos para percibir situaciones de despilfarro y devaluación de los recursos naturales. Con el agua sigue siendo la misma, y en teoría la idea de preservar este recurso a pesar de ser algo enfatizado, en la práctica no hay 
una gran movilización para prevenir consecuencias previsibles de un futuro con escasez de agua. La siguiente cita define claramente la situación en cuestión:

Uno de los principales retos de la sociedad en este siglo XXI se refiere a la urgente necesidad de preservar los recursos hídricos en calidad y cantidad, en un escenario de aumento poblacional y baja sostenibilidad de los procesos de producción y consumo.

[...]Estas previsiones de crecimiento poblacional y estimaciones vinculadas a la producción, conservación y distribución de alimentos consideran que si la población mundial alcanza los 10 mil millones de habitantes en los próximos 50 años, tendremos el $70 \%$ de los habitantes del planeta Abordar las deficiencias en el suministro de agua, afectando a alrededor de 1.06 mil millones de personas que no tendrán ni siquiera agua para alimentos básicos (SILVA, 2009, p. 1).

Si bien en muchos países del mundo el agua ya se ha convertido en una disputa que genera diversos conflictos, Brasil aún no se ha dado cuenta de la gran responsabilidad que tenemos con la calidad de vida de las generaciones futuras. Según Célia Jurema Aito, Brasil es un país privilegiado en términos de disponibilidad de agua, ya que cuenta con el $28 \%$ de la disponibilidad sudamericana y el $12 \%$ de las reservas de agua del mundo. En territorio brasileño, el $72 \%$ del agua se encuentra en la cuenca amazónica. El río Amazonas tiene 6.885 kilómetros de extensión y es el más grande del mundo en volumen de agua, vertiendo 175 millones de litros por segundo en el Océano Atlántico. Sin embargo, no se puede olvidar que el crecimiento de la población hace que el riesgo de escasez llegue a nosotros también. Según Célia Jurema, entre 1970 y 2000, Brasil pasó de ser una población urbana del 55\% al 82\% de la población total. Se sabe que más de 1.400 millones de personas no tienen acceso al agua potable y otros 2.000 millones no tienen ningún tipo de saneamiento básico.

A medida que el hombre moderno evolucionó en tecnología, dejó de tener contacto con su esencia natural, pasó a vivir cada vez más lejos de su base, la naturaleza, y convertirse en un ser ansioso e irritado. El desequilibrio colectivo al que se enfrenta 
nuestro planeta representa profundamente un desequilibrio de los individuos que componen la población mundial. Este desequilibrio se relaciona con un fracaso en el proceso de evolución del hombre que se distanció de sus orígenes, busca, la mayoría de las veces, su bienestar individual, olvidando que somos parte de un organismo vivo llamado "sociedad" y para que Desarrollar eficazmente, es necesario asegurar el bien de todos y no sólo de uno mismo. En las grandes ciudades, se percibe que cada vez más personas se sienten solas, indefensas, incluso viviendo en medio de la multitud, lo que las hace más individualistas y egoístas cada día. Y aunque hoy en día, existe una idea de conservación, al menos en los anuncios de muchas empresas que pretenden izar la bandera de la preservación de un "marketing empresarial" que sea rentable, lo gran cierto es que poco hacer para la preservación del medio ambiente y En consecuencia, el agua en el país. Por lo tanto, sentimos la ineficiencia, a pesar de tantos esfuerzos, de nuestros gobernantes, de las organizaciones no gubernamentales e incluso de la propia población en relación con la cuestión ambiental, ya que todo el trabajo se hace básicamente para resolver los problemas Cuando el proceso de cambio y mejora debe comenzar en el nivel interno de cada persona.

Desafortunadamente, la población mundial que ocupa cada vez más espacio en el planeta es directamente responsable de la degradación de los ríos, las zonas costeras, los océanos, las playas y, como esta población no crece, el volumen de basura se ha hecho más grande cada día. Para hacerse una idea del problema, sólo los estadounidenses producen 212 millones de toneladas de basura al año y mueven casi 3 millones de botellas de plástico por hora. Se necesita una conciencia para deshacerse de este tipo de basura en lugares para el reciclaje. En Brasil, la política nacional de residuos sólidos (Ley Federal 12.305/2010) determina que todo el material producido por actividades domésticas y comerciales que será posible recoger por los servicios de limpieza pública, debe ser remitido al destino Finalizar sólo cuando no sea posible reutilizarlo, ya sea a través del reciclaje, la reutilización, el compostaje o la generación de energía. Cuando no exista tecnología viable, los residuos deben destinarse a vertederos. Sin embargo, del volumen producido en 2016, casi 30 millones de toneladas no tenían el destino adecuado, lo que representa un porcentaje 
del $41,6 \%$ del total generado, lo que transforma los residuos sólidos en un grave problema ambiental, porque la eliminación inadecuada puede Cargando este material en arroyos y ríos y, en consecuencia, llega a los océanos, además del problema de los vertederos, los impactos en la salud pública entre otros efectos negativos.

En promedio se producen 80 millones de toneladas de residuos sólidos urbanos, siendo casi 470 kilos por habitante, donde el $60 \%$ de este total es material reciclable. Sin embargo, sólo el $3 \%$ de la basura producida se reenvía para su reciclaje.

La impresión es que en todas partes las aguas superficiales están siendo contaminadas con una aterradora variedad de residuos urbanos, industriales y agrícolas. Incluso en los países industrializados, donde existe legislación sobre la calidad del agua, la contaminación sigue siendo un problema que molesta. En los Estados Unidos, se encontró que el $60 \%$ de los lagos encuestados están contaminados por nutrientes de fertilizantes y estiércol animal, lo que está causando la muerte casi masiva de peces, además de la degradación de la calidad del agua. En Canadá, 20000 lagos son golpeados por lluvias ácidas.

El crecimiento demográfico y económico de Brasil en los últimos años ha utilizado recursos hídricos mucho más allá de su capacidad para apoyar tanto la cantidad como la calidad. Los graves problemas de gestión del agua son causados por la expansión desordenada de las poblaciones urbanas y la limitada disponibilidad de recursos hídricos, además, las políticas públicas encaminadas a preservar las aguas son insuficientes Demanda de consumo y utilización. La ocupación de los espacios llanos, cerca de los ríos y sin control, causado durante años y años, pequeñas inundaciones en un gran número de ciudades en el sur y sureste, en Brasil. En los años 80, se produjeron varias inundaciones junto al río y con grandes pérdidas, principalmente en Paraná, Santa Catarina, Sao Paulo y Minas Gerais. En nuestro país hay una gran diversidad de situaciones, con muchos recursos hídricos en las regiones norte y medio oeste y escasez en las regiones noreste y sureste, en oposición a la mayor concentración de la demanda, junto con la región sur. El desarrollo urbano también aumenta las áreas resistentes al agua y de baja absorción a través de residencias, paseos, calles, estacionamientos e incluso parques. Dado que el agua de lluvia no 
puede infiltrarse en el suelo, el volumen adicional drena en el sistema de drenaje hacia los ríos. En la década de los 70, había una gran esperanza de que fuera posible planificar el crecimiento de las ciudades y planificar su futuro armónicamente. Sin embargo, 37 años después, parece que la planificación urbana en Brasil, o incluso en el extranjero, fue atrofiada lo que llevó a la acumulación de problemas.

El paseo marítimo brasileño es víctima de la furia inmobiliaria, especialmente en Río de Janeiro, además de las playas del noreste y del sur. El crecimiento rampante proporciona la aparición de aldeas y favelas donde no se encuentra agua potable y, mucho menos, aguas residuales. Río de Janeiro y Sao Paulo, las dos ciudades brasileñas más grandes, tienen graves déficits de red de tratamiento de aguas residuales $\mathrm{y}$, a pesar de tener préstamos internacionales, no reducen el alto nivel de contaminación de la bahía de Guanabara y el río Tietá, este Debido a la falta de conciencia de la población y un programa de educación ambiental que estimula los cambios en el comportamiento de las personas.

Las ciudades más grandes del país administran mal sus recursos hídricos. En 1997, el Banco Mundial ofreció 40.000 millones de dólares EE.UU. para empresas de saneamiento. Casi el 100\% del dinero sigue intacto porque las empresas brasileñas no están bajo los requisitos técnicos mínimos para recibir préstamos-afirmación del geólogo Aldo Rebou-as, de la empresa de Investigación de Recursos Minerales - esto porque el banco Determina que las empresas tienen una pérdida máxima del $20 \%$ en las redes de distribución de agua. Sólo en Sao Paulo, en 2001, las pérdidas fueron del $30 \%$.

La degradación ambiental es uno de los aspectos más críticos del proceso de deterioro causado directa e indirectamente por el hombre. Las regiones que antes tenían cantidades de recursos hídricos, hoy comienzan a dar señales de escasez, y la explicación es el residuo con explotación excesiva, el silting de los ríos y la contaminación de las fuentes. $Y$ todos estos problemas se originan casi siempre en la explosión de la agricultura industrial que sirve para alimentar a los millones de habitantes de las ciudades. Es un hecho que la urbanización y el crecimiento 
desenfrenado de las ciudades aumentan significativamente el consumo y el mal uso del agua.

\section{¿QUE HACER EN CARA DE TAL RESIDUO?}

La descontaminación del agua no es sólo un problema técnico, sino también de orden económico y social, porque no hay recursos suficientes para implantar los sistemas de purificación de aguas residuales líquidas con la tecnología disponible. Se sabe que los estados han sido capaces de capturar recursos a través de bancos internacionales y dar los primeros pasos a favor de nuestras fuentes, como en Rio Grande do Sul, con el Pró-Guaíba (programa estatal) y Guaíba Vive, en la capital Porto Alegre, como Referencia al problema de los recursos hídricos. Sin embargo, aún queda mucho por hacer, porque se necesita agua en todos los aspectos de la vida. Los recursos de agua dulce constituyen un componente esencial de la hidrosfera de la Tierra y una parte indispensable de todos los ecosistemas terrestres, y su escasez generalizada, destrucción gradual y aumento de la contaminación de las fuentes de agua en muchas regiones del mundo Exigir conciencia y cambios en las actitudes hacia el agua. Y mientras que muchos desperdician y desperdician, otros ya sufren las consecuencias de la escasez de agua. La desigualdad de distribución y los desechos son dos razones fuertes que explican en parte por qué 1.400 millones de personas —casi cinco veces la población de los Estados Unidos- no tienen acceso al agua potable, y el agua es un factor principal para la salud. También se sabe que el $60 \%$ de los recursos hídricos están situados en sólo nueve países, entre ellos Brasil, pero son 80 países que representan el $40 \%$ de la población mundial, que sufren de escasez de agua. Sin embargo, esto no significa que los Estados Unidos, Brasil, Rusia, Sudáfrica o China, considerados países ricos en agua, no estén libres de problemas de falta de agua.

En cuanto a los residuos, según Rodolfo F. Alves Pena, Agricultura es responsable de la absorción en promedio en todo el mundo, el $70 \%$ de los suministros de agua, y este índice va al 80 a $90 \%$ en los países subdesarrollados. El agua potable se pierde en promedio un $50 \%$ en las fugas de los sistemas de distribución y si no se hace nada en el sentido de los cambios de comportamiento, en el año 2025 habrá más de 4 mil millones de personas que no tendrán acceso a este precioso y esencial para la vida. 
Por lo tanto, es necesario analizar la cuestión de los no residuos de agua en el medio rural de forma integrada, en la que varios factores influyen en el cambio en el hábito de los productores, en el caso del uso adecuado de los recursos hídricos, como explica Oliveira (2008):

La decisión y la opción para el uso más eficiente de los recursos naturales en general y del agua en particular deben darse de manera integrada y asociadas con los instrumentos de gestión en todos los niveles pertinentes. Sólo así será posible alcanzar un nivel que permita una adecuación de la oferta y la demanda con vistas al uso racional de un recurso tan valioso. (OLIVEIRA, 2008, p.3)

Esta conciencia debe ocurrir en los diversos ámbitos de la sociedad, independientemente de la condición social, cultural e intelectual de las personas. Esto se debe a que hoy en día, un ciudadano europeo consume 150 litros de agua al día, mientras que un indio tiene que conformarse con sólo 25 litros al día. Un estadounidense consume 3.000 litros de agua al día.

Es bueno destacar que el desperdicio del papel es más un agravante en la tala de árboles, así como el consumo de agua, ya que, para producir una tonelada de fibra virgen, es necesario 44000 a 83000 litros de agua. Es necesario fomentar el uso de papel reciclado, ya que, por cada tonelada de papel utilizado, se puede producir casi una tonelada de papel nuevo (más eficiente que la proporción de 2-3,5 toneladas de árboles para producir una tonelada de papel virgen). Debe estimularse el proceso de educación ambiental.

El agua dulce, un recurso finito, es altamente vulnerable y de usos múltiples, por lo que debe gestionarse de manera integrada con mecanismos eficaces de coordinación e implementación. En los planes para su uso, es necesario destacar la protección, conservación y gestión sostenible y racional basada en las necesidades y prioridades de cada comunidad. La evaluación de los recursos hídricos es la base práctica para su gestión integrada y sostenible, así como un examen equilibrado de las necesidades de la población y el medio ambiente. 
Por todas las razones aquí expuestas, es necesario tener en cuenta que el agua es el bien más preciado de este siglo, esto, sin embargo, no significa que se deba transformar la "fuente de vida" en un sistema monetario, generador de tantos conflictos, sino más bien unir esfuerzos a favor consumo consciente y la preservación de este recurso vital para garantizar la calidad de vida de las generaciones futuras.

\section{CONSIDERACIONES FINALES}

La sostenibilidad es la relación entre los sistemas de economía humana y el medio ambiente. Las acciones de los hombres deben estar en sintonía con el medio ambiente, garantizando el mantenimiento y la preservación para mantener la diversidad, complejidad y funcionamiento del sistema ecológico que nos mantienen vivos. Y pensar de manera sostenible es necesario, porque los recursos naturales son finitos. Además, la calidad de los recursos se ha empeorado cada vez más debido al aumento de la población y a la falta de políticas públicas destinadas a preservarlos. Como ejemplo, se estima que aproximadamente 12 millones de personas mueren anualmente debido a problemas relacionados con la calidad del agua.

La única manera en que la mayoría de los países pueden tratar la creciente contaminación de sus recursos hídricos es la implementación de estrategias de gestión integradas, que no sólo ayudan a limpiar los cursos D' Agua, sino que, en primer lugar, también previenen la contaminación. Hasta ahora, muy pocos países han podido practicar esos cambios. Tienes que estar alerta. Tendremos que evitar los residuos, interrumpir los procesos contaminantes y crear nuevas formas de controlar, capturar y distribuir.

Se necesita una respuesta cultural y ética. La situación por la que pasa el mundo, no es más que un estilo de vida más sofisticado, donde lo que uno quiere "debe tener", causando así la aceleración de las fábricas e industrias que se encuentran entre los mayores usuarios de agua que sólo pierden por la agricultura y el uso del poliéster Plástico. En el siglo TWENTIETH la población creció tres veces, mientras que el consumo de agua aumentó en seis. Incluso nosotros los brasileños, que tenemos el $16 \%$ de toda el agua en el mundo, estamos sufriendo de racionamientos debido a la 
mala administración de los recursos y la infraestructura inadecuada. Los países áridos se enfrentan a desafíos más aterradores como el Oriente Medio, donde hay áreas donde la escasez limita el crecimiento socioeconómico.

Las medidas conjuntas de gestión para el uso de la tierra y los recursos hídricos deben ser más frecuentes a fin de aumentar la eficiencia del uso del agua de riego, evitando así la erosión del suelo, las inundaciones, la sedimentación, la salinización y los efectos nocivos de Plaguicidas y fertilizantes en organismos. Además, la degradación ambiental ha generado una patología ambiental emergente, y enfermedades como el cólera y el dengue se han vuelto más causadas con fuerza por la contaminación del aire, el agua y los suelos, así como por el uso de sustancias tóxicas y peligrosas, como Plaguicidas y otros productos de cuidado especial. Como hemos visto hasta ahora, la degradación no es sólo del medio ambiente, sino también del ser humano, cuyos valores se pierden, donde las necesidades ya no son las básicas, y más bien las superfluas, donde el mal es el correcto. Aquí es donde veremos la educación ambiental como una prioridad para alcanzar los objetivos del desarrollo sostenible. La conferencia de Tiflis celebrada en 1977, que se ocupa de la educación ambiental a nivel internacional, hace que ver a la EA sea la formación de una conciencia fundada en una nueva ética que resistirá toda explotación. Esto es lo que la humanidad necesita, dar un giro en sus acciones, en sus inversiones, en sus actitudes, en sus valores, para darse cuenta de que no sólo está pensando en sí mismo que será capaz de dar un futuro saludable a sus generaciones.

Sabemos que el hombre es el principal causante de tanto desequilibrio. El hombre perturbaba tan completamente el entorno natural donde se movía que ya no conoce su lugar en este sistema, excepto, por supuesto, como un factor de trastorno. Hasta ahora, nuestros intentos de dominar el medio consistían en simples reformas paisajísticas, llevadas a cabo torpes e irreveriosamente; Mientras que otras actividades humanas han producido efectos secundarios nocivos, imprevistos y mal entendidos. Todos los océanos han sido contaminados por los seres humanos, la atmósfera e incluso las capas glaciares remotas. La mayoría de los ríos están más o menos contaminados y muchos de ellos, como el río Tiet y el Tíber, se transformaron 
en aguas residuales al aire libre. Barbie Nadeau, periodista de Newsweek en 2002, estuvo en Roma y da su testimonio sobre el Tíber "... Flotan peces muertos diezmados, no conocidos por qué. El hedor está en todas partes, un residuo aceitoso hace que las anguilas y las carpas muertas brillen "y concluya:"... Si un río es la línea simbólica de la vida de una ciudad, entonces Roma está en una situación bastante mala. " El caso no es dominar el medio, sino saber si la naturaleza puede ser preservada con cierta apariencia de orden y si la civilización es capaz de sobrevivir a la transformación que provoca en la naturaleza. La evolución cultural nos ha llevado gradualmente a reconocer que la humanización del planeta sólo puede tener éxito si se respetan las leyes ecológicas fundamentales.

Es hora de empezar a pensar en cambiar hábitos. Sentir que formamos juntos una sola cadena; Si rompes un eslabón, la cadena no tendrá más sentido. Todo el mundo necesita a todos. Los eruditos indios, hace miles de años, ya dijeron que el todo está contenido en las partes $y$, por mucho que las cosas parezcan diversificadas y plurales, de hecho, son aspectos de la misma y única realidad. Aquí hay una frase a mediar, por todos nosotros, para que podamos entender la dinámica de la vida y ver que no hay yo y sí, los nudos. Hasta ahora la naturaleza se ha utilizado de forma gratuita, y gracias a ella producimos y vivimos. Sin embargo, en el panorama del crecimiento de la población mundial no ha reconocido su debido valor.

En relación con el regadío, responsable de los residuos y el consiguiente compromiso de los manantiales, para minimizar los impactos de su uso inadecuado, Rebous (2001) destaca que es necesario una planificación más eficiente y racional del uso del riego y la optimización del equipo utilizado. También puede reducir los costos de electricidad.

Otra práctica reciente en Brasil y que ha ido ganando fuerza es el uso de agua de reutilización para abastecer la necesidad de irrigación de diferentes cultivos alimentarios. Hespanhol (2002) complementa que en varios países en los que se utiliza esta técnica, la productividad aumenta significativamente cuando está bien planificada. 
Cualquier forma de uso eficiente del agua destinada a minimizar los residuos en el campo depende directamente de la planificación realizada por el productor. La planificación es indispensable para armonizar los diversos usos del agua, permitiendo a los diferentes sectores productivos, monitoreando la cantidad y calidad de los recursos hídricos, mejorando los niveles de eficiencia general de uso (PAZ et al., 2000).

Segundo (FURRIELA,2001) El primer gran desafío que surge no sólo para el productor rural, sino también para toda la humanidad es la conciencia de que no hay suministro inagotable de agua potable en el planeta. Deberían promoverse algunas medidas para garantizar el suministro de agua en cantidad y normas aceptables. El gran desafío de hoy en día es reducir el nivel de degradación del agua mediante medidas como la conservación, la mejora del saneamiento básico, la reducción del uso de plaguicidas, la producción industrial más limpia y la gestión del consumo.

Cada día es más evidente la necesidad de percibir el agua como un bien económico, en el que su gestión debe basarse en principios de eficiencia económica, de una manera que satisfaga la demanda desde una perspectiva de sostenibilidad.

Añade también que es necesario establecer una nueva forma de pensar y actuar, adoptando diferentes hábitos, usos y costumbres, donde el objetivo general es el crecimiento económico, basado en el consumo sostenible de agua, promoviendo así la protección de Manantiales aún conservados y la recuperación de los ya perjudicados, señala que esto no es suficiente, y es necesario hacer mucho más para lograr este objetivo debe, por lo tanto, tratar el agua como una prioridad social y ambiental, ya que es necesario Cuidar de los recursos hídricos brasileños, ya que la creciente necesidad de agua mundial traerá al país la posibilidad de aprovechar su crecimiento y desarrollo, y de mantener un aporte esencial a la vida y a cualquier actividad productiva. 


\section{REFERENCIAS BIBLIOGRÁFICAS}

AGENDA 21. The Earth Summit Strategy to save our Planet. Ed. Daniel Sitarz, Earthpress, 321p. Boulder Colorado, 1994.

AGÊNCIA NACIONAL DAS ÁGUAS. Disponível em: <www.ana.gov.br> Acesso em: $20 / 07 / 2019$

Baía de todas as águas - Preservação e Gerenciamento Ambiental na Bacia Hidrográfica do Guaíba - Pró-Guaíba, RS/1998.

EBC. Disponível em: <www.ebc.com.br/.../mais-de-75-das-florestas-primarias-domundo-nao-existem-mais >Acesso em:19/07/2019

FURRIELA, R. B. EDUCAÇÃO PARA O CONSUMO SUSTENTÁVEL. Ciclo de Palestras sobre Meio Ambiente - Programa Conheça a Educação do Cibec/InepMEC/SEF/COEA, 2001.

HESPANHOL, I. Potencial de Reuso da Água No Brasil, Agricultura, Industria, Municípios, Recarga de Aquíferos. RBRH- Revista Brasileira Dos Recursos Hídricos. Vol. 7 n. 4, 2002

JUSBRASIL. Disponível em: <https://www.jusbrasil.com.br/topicos/10603350/artigo90-do-decreto-n-24643-de-10-de-julho-de-1934> Acesso em 20/07/2019

MUNDO

DA

EDUCAÇÃO.

Disponível

em:

$<$ https://mundoeducacao.bol.uol.com.br/geografia/consumo-agua-no-mundo.htm> Acesso em 12 de setembro de 2018.

OLIVEIRA, E. C. A Gestão Dos Recursos Hídricos Sob a Perspectiva da Descentralização. ANAP BRASIL - REVISTA CIENTÍFICA, ano 1 no 1, 2008.

PORTAL FMU. Disponível em: <https://portal.fmu.br/reciclagem-no-brasil-panoramaatual-e-desafios-para-o-futuro/> Acesso em: 20/07/2019 
PUC

RS.

Disponível

em:

$<$ www.pucrs.br/edipucrs/online/planetaagua/planetaagua.pdf>

Acesso

em:

$20 / 07 / 2019$

REVISTA EDUCAÇÃO AMBIENTAL EM AÇÃO. Disponível em: <http://www.revistaea.org/artigo.php?idartigo=1271> Acesso em: 17/07/2019

REBOUÇAS, A. C. Água e desenvolvimento rural. ESTUDOS AVANÇADOS, 2001.

REBOUÇAS, A.C. Água no Brasil: Abundância, Desperdício e Escassez.BAHIASalvador, v. 13, n. ESPECIAL, p. 341-345, 2003 ANÁLISE \& DADOS

SILVA,J. S. Água e Agricultura Irrigada. Palestra-Seminário Agricultura Irrigada Com Desenvolvimento Sustentável-Universidade Federal De Uberaba-Em 24-09-2009. Disponível

em:

http:www.irrigacao.org.brdocdownloadAgua\%20Agricultura\%20Irrigada_JoseSilverio. pdf.. Acessado em: 20/07/2019

TUCCI, C. E. M. Gestão da água no Brasil. Brasília: UNESCO, 2001. 156p.(1. Hidrologia - Brasil II. Hespanhol, Ivanildo III. Cordeiro Netto,

VICTORINO, C. J. A. Planeta água morrendo de sede: uma visão analítica na metodologia do uso e abuso dos recursos hídricos. Porto Alegre: EDIPUCRS, 2007.231 p. (1. Água - Uso. 2. Água - Qualidade. 3. Recursos).

Enviado: Junio de 2019.

Aprobado: Agosto, 2019. 